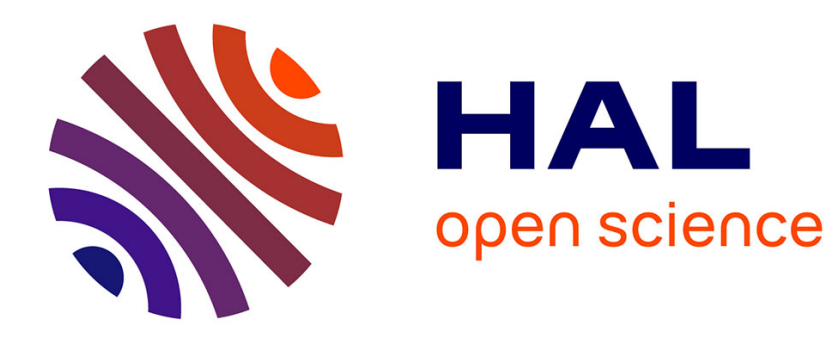

\title{
La bonne distance. L'idéologie de la complémentarité légitimée en centres de loisirs
}

\author{
Elisa Herman
}

\section{To cite this version:}

Elisa Herman. La bonne distance. L'idéologie de la complémentarité légitimée en centres de loisirs. Cahiers du Genre, 2007, 42 (1), 10.3917/cdge.042.0121 . halshs-01674946

\section{HAL Id: halshs-01674946 \\ https://shs.hal.science/halshs-01674946}

Submitted on 3 Jan 2018

HAL is a multi-disciplinary open access archive for the deposit and dissemination of scientific research documents, whether they are published or not. The documents may come from teaching and research institutions in France or abroad, or from public or private research centers.
L'archive ouverte pluridisciplinaire HAL, est destinée au dépôt et à la diffusion de documents scientifiques de niveau recherche, publiés ou non, émanant des établissements d'enseignement et de recherche français ou étrangers, des laboratoires publics ou privés. 


\title{
LA BONNE DISTANCE. L'IDÉOLOGIE DE LA COMPLÉMENTARITÉ LÉGITIMÉE EN CENTRES DE LOISIRS
}

\author{
Elisa Herman
}

L'Harmattan | Cahiers du Genre

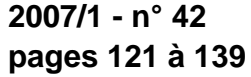

\section{ISSN 1298-6046}

Article disponible en ligne à l'adresse:

http://www.cairn.info/revue-cahiers-du-genre-2007-1-page-121.htm

Pour citer cet article :

Herman Elisa, « La bonne distance. L'idéologie de la complémentarité légitimée en centres de loisirs », Cahiers du Genre, 2007/1 n 42, p. 121-139. DOI : 10.3917/cdge.042.0121

Distribution électronique Cairn.info pour L'Harmattan.

(c) L'Harmattan. Tous droits réservés pour tous pays.

La reproduction ou représentation de cet article, notamment par photocopie, n'est autorisée que dans les limites des conditions générales d'utilisation du site ou, le cas échéant, des conditions générales de la licence souscrite par votre établissement. Toute autre reproduction ou représentation, en tout ou partie, sous quelque forme et de quelque manière que ce soit, est interdite sauf accord préalable et écrit de l'éditeur, en dehors des cas prévus par la législation en vigueur en France. II est précisé que son stockage dans une base de données est également interdit. 


\title{
La bonne distance. L'idéologie de la complémentarité légitimée en centres de loisirs
}

\section{Elisa Herman}

\begin{abstract}
Résumé
Cette enquête en centres de loisirs montre ce que l'organisation des équipes d'animatrices et d'animateurs doit conjointement au soupçon de pédophilie pesant plus facilement sur les hommes, et à l'assignation des femmes au travail de care dans le cadre de la division sexuée du travail. Dans un contexte médiatique de 'panique morale' à ce sujet, on observe une légitimation accrue de l’idéologie de la complémentarité, tandis que l'éventualité d'une interchangeabilité des animatrices et des animateurs devient moins probable. Cela conduit à un renforcement des assignations de genre et de la hiérarchie des tâches selon leur proximité avec le domaine du sale et du soupçon, et introduit une inégalité entre animatrices et animateurs face à ces situations. ${ }^{1}$

CORPS - CARE - CENTRES DE LOISIRS - PETITE ENFANCE - RÔLES SEXUÉS — MIXITÉ PROFESSIONNELLE — PÉDOPHILIE
\end{abstract}

\footnotetext{
${ }^{1}$ Cet article présente un travail s'appuyant sur une note de recherche réalisée sous la direction de Régine Sirota, et sur l'enquête effectuée en maîtrise et en master 2 de sociologie sous la direction de François de Singly. Les noms de lieux et de personnes ont été modifiés afin de préserver l’anonymat des enquêté(e)s. Je remercie sincèrement Régine Sirota, Olivier Schwartz et François de Singly pour leurs remarques sur les différents volets de cette recherche. Cet article doit aussi beaucoup aux réflexions suggérées par Danièle Kergoat et par Liane Mozère, que je tiens à remercier. Enfin, un grand merci à Marc Bessin, à Numa Murard et aux participants de leur séminaire pour leur intérêt et la qualité de la discussion lors de la présentation de cette recherche.
} 
Les centres de loisirs sans hébergement (CLSH) sont des institutions périscolaires où de jeunes adultes munis du BAFA ${ }^{2}$, et le plus souvent étudiants, accueillent des enfants scolarisés de 2 à 11 ans, lors des mercredis et des vacances scolaires. Ce travail, réalisé au sein de deux centres de loisirs maternels et primaire des Hauts-de-Seine, s'appuie sur une enquête ethnographique fondée sur l'observation participante et une vingtaine d'entretiens approfondis avec les membres de l'équipe d'animation. Les deux structures étudiées sont assez différentes par leur taille et leur organisation, cependant la façon dont les animatrices et animateurs sont répartis selon l'âge des enfants, puis selon les activités et les tâches données, semble relativement typique de la «sexuation du travail social» (Bessin 2005). À Jules Ferry, environ cent trente enfants de 3 à 11 ans sont accueillis par une équipe de trente et une personnes, soit d'une part dix animatrices et six animateurs pour les enfants de maternelle, et d'autre part huit animatrices et sept animateurs pour les enfants d'élémentaire. À la Colline, la répartition est équivalente, seulement soixante enfants de 3 à 11 ans sont accueillis chaque mercredi par deux animateurs et six animatrices pour les plus petits, et pour les plus grands par cinq animateurs et cinq animatrices.

Cet article propose de s'interroger sur la manière dont les animatrices et les animateurs se répartissent le travail d'animation au sein des équipes. Le centre de loisirs maternel sera au centre de cette analyse, car le travail de care ${ }^{3}$ auprès des enfants plus jeunes est plus intense. Dans ce contexte, ce travail peut être identifié à du 'maternage' (comme le font certains enquêtés) et a pour objectif de construire l'autonomie de l'enfant tout en reconnaissant sa vulnérabilité et sa dépendance envers l'adulte. C'est particulièrement dans l'exercice de cette 'compétence' qu'on peut s'interroger sur la place des hommes et des femmes dans l'équipe, quand la question de la 'panique

\footnotetext{
${ }^{2}$ Brevet d'aptitude aux fonctions d'animateur : 'diplôme non professionnel' indispensable pour travailler en centre de loisirs, s'obtenant dès 17 ans.

${ }^{3}$ Le care se définit comme une disposition morale et pratique à l'attention envers autrui, au soin, à l'affection et à la proximité.
} 
morale' autour de la pédophilie ${ }^{4}$ fait planer sur chacun — et surtout sur les hommes - un soupçon déstabilisant.

La notion de 'panique morale', élaborée par Erich Goode et Nachman Ben-Yehuda (1994) à partir du travail pionnier de Stanley Cohen (1972), désigne à la fois la présence de médias qui vont intensifier une peur autour d'un problème social, et une disproportion entre l'angoisse collective et la menace présente. Il s'agit donc des représentations d'un problème social développées par les enquêtés, qui se manifestent par des récits et des normes utilisés pour éloigner le soupçon pouvant peser principalement sur les hommes s'occupant d'enfants. Cette notion semble intéressante face au vocabulaire de la prise de risque et de la précaution, parce qu'elle évoque précisément le domaine des émotions, des ressentis, de la réflexivité par rapport au travail. S'il s'agit d'une 'panique', c'est que les enquêtés observés confient leur peur d'être mal jugés. Quant au terme 'moral', il renvoie au caractère non quantifiable scientifiquement de cette menace, qui est avant tout présente dans les esprits, bien que pouvant se matérialiser dans les corps.

Pour comprendre la façon dont hommes et femmes s'organisent dans les équipes étudiées, on a choisi de condenser en deux expressions deux modes d'organisation antagonistes: l'idéologie de la complémentarité sexuée s'opposant à l'aspiration à l'interchangeabilité entre animatrices et animateurs. On présentera d'abord un état des lieux statistique de la présence des hommes et des femmes dans l'animation, puis la manière dont celui-ci est justifié par l'idéologie de la complémentarité dans les propos des animateurs et animatrices interrogés. Cela conduira à détailler les conditions d'émergence d'équipes tota-

\footnotetext{
${ }^{4}$ Dans le contexte du centre de loisirs, et plus généralement des institutions de prise en charge de la petite enfance, les représentations véhiculées par les personnes rencontrées au sujet de la 'pédophilie' définissent cette notion comme des attitudes corporelles et des gestes 'inappropriés', au sens où ils peuvent blesser l'enfant dans son intimité et son intégrité corporelle. Cette notion de pédophilie est bien sûr socialement et historiquement construite, apparaissant avec cette intensité médiatique depuis quelques années seulement, cependant cet aspect n'est pas au centre de l'article, qui s'attache à montrer comment le soupçon de gestes 'déplacés' (touchant l'enfant d'une façon interprétée comme sexuelle) contribue sur les terrains observés à façonner les rapports sociaux de sexe dans l'équipe, au même titre que la division du travail plus ancienne.
} 
lement mixtes (rassemblant autant de femmes que d'hommes) face au phénomène actuel de panique morale, qui peut amener les hommes travaillant auprès de jeunes enfants à éviter certaines pratiques (celles du travail de care : les câlins, et les moments de soin et d'hygiène) qui font pourtant partie des actes professionnels prescrits. Alors que les animateurs et animatrices travaillaient déjà sur le mode de la complémentarité sexuée, en naturalisant les compétences mises en œuvre, cette idéologie s'est vue renforcée par la panique morale, permettant de justifier la répartition différenciée : aux femmes les activités manuelles et le travail de care, aux hommes les activités physiques et sportives et l'autorité.

\section{Idéologie de la complémentarité : une naturalisation justi-} fiant la place des femmes en centres de loisirs maternels

\section{Une majorité de femmes en centres de loisirs}

Les équipes d'animation étudiées correspondent à la répartition fréquente dans l'animation, où la moyenne nationale compte $63 \%$ de femmes contre $37 \%$ d'hommes pour l'animation socioculturelle, quand les crèches et les garderies comptent $92 \%$ d'emplois féminins ${ }^{5}$ (Rousseau 1997, p. 211). S’il est difficile d'évaluer le nombre d'animateurs BAFA en exercice en centres de loisirs, on sait néanmoins que le nombre de BAFA délivrés en 2004 est d'environ 9500 en Île-de-France, dont plus des deux tiers à des femmes ${ }^{6}$. Les femmes sont plus nombreuses en centres de loisirs maternels, et plus souvent assignées aux tâches du travail de care. Cette assignation naturalisée constitue le fondement de l'idéologie de la complémentarité entre les sexes.

On voit que l'obstacle à une mixité totale de la profession tient à la représentation du contenu de l'activité : la disposition au travail de care étant considérée comme un attribut de genre imparti aux femmes plus qu'aux hommes. Or, si une majorité des enquêté(e)s se dit favorable à des équipes complètement

\footnotetext{
${ }^{5}$ Source : Unedic au 31/12/1993.

${ }^{6}$ Source : Direction régionale de la jeunesse et des sports d'Île-de-France.
} 
mixtes (exactement autant d'hommes que de femmes), aucun n'est favorable à des équipes majoritairement masculines, ce qui souligne l'importance de l'opposition construite entre virilité et féminité dans le soin aux enfants. C’est également le phénomène de la panique morale exposant davantage les hommes au soupçon de la pédophilie, qui s'exprime ici selon les enquêté(e)s. Parfois, comme l'explique une animatrice, il existe une interdiction explicite pour les animateurs de pratiquer certains gestes ou tout du moins de le faire sans la présence d'une femme, interdiction formulée par la directrice d'un centre de loisirs croyant qu'il existe une loi à ce sujet, ce qui n'est pas le cas.

\section{Complémentarité ou interchangeabilité entre animateurs et animatrices au niveau du travail de care?}

Plus les enfants sont âgés, plus la présence des hommes augmente à leurs côtés. Cette coprésence des hommes et des femmes en centres de loisirs maternels, s'apparente à une 'mixité sexuée’ (Bacou 2004) et n’est pas pour autant une interchangeabilité des animateurs et animatrices vis-à-vis des tâches auxquelles ils sont confrontés. Magali Bacou constate également une répartition sexuée des activités et des attitudes (il faudrait dire genrée, car elle se base sur une construction sociale de compétences acquises). La mixité dans l'équipe pose la question de la répartition des tâches, or "c'est parce que la mixité est un construit social reposant sur la conception différenciée des genres qu'elle ne rime pas avec égalité » (id.). Effectivement, cela se traduit par un partenariat entre hommes et femmes au sein de l'équipe d'animation à la manière d'une complémentarité et non d'une interchangeabilité, bien que cette dernière soit parfois souhaitée par des enquêtées. L’idée d'une interchangeabilité entre les personnes et d'une ressemblance dépassant la logique différenciatrice du genre est parfois évoquée par les animateurs et animatrices interrogés. Cette perspective minoritaire s’éloigne de l’idéologie de la complémentarité des différences sexuées qui est une manière d'enfermer les attributs de genre dans un statut biologique, sans offrir le moyen de variations individuelles, qui sont pourtant observables au centre de loisirs. Dans l'aspiration à l'interchangeabilité revendiquée 
par certain(e)s enquêté(e)s, les qualités d'autorité et de care sont pensées comme asexuées. Cependant, la logique de la complémentarité semble la plus présente au sein des équipes étudiées, où la différenciation entre animateurs et animatrices permet aux enquêté(e)s d'asseoir des places qui sont assignées et revendiquées (les critiques à ce sujet étant minoritaires).

Joachim (20 ans, animateur en CLSH maternel chez les toutpetits) associe implicitement les qualités de care et d'autorité à des attributs de genre, en les naturalisant, et présente une justification récurrente de l'idéologie de la complémentarité où l'importance du nombre de femmes est légitimée par le fait que l'autorité n'est pas à ce point nécessaire auprès des enfants, et que l'harmonie dans l'équipe serait perdue si celle-ci était davantage mixte :

On va dire, en partant du principe qu'un homme est plus autoritaire qu'une femme, ce qui n'est pas forcément vrai [sourire], qu'on est suffisamment de garçons. Parce que, enfin c'est difficile à dire mais il y a un certain équilibre entre les animatrices et les animateurs, et bon ça se passerait beaucoup moins bien s'il y avait plus de garçons, voilà...

Les critères de distinction entre les personnes qui seront perçues comme aptes ou non à agir dans telle ou telle situation tendent à se restreindre au sexe de l'individu, conduisant à se demander si « après l'ancienne opposition laïque/confessionnel, la distinction femme/homme (dedans/dehors, maternel/primaire, etc.) ne reste pas la référence identitaire la plus stable » (Lebon 2005) permettant d'asseoir des légitimités à agir hiérarchisées et délimitées par le genre. De plus, la Convention interministérielle pour la promotion de l'égalité des chances entre les filles et les garçons, les femmes et les hommes dans le système éducatif ${ }^{7}$ semble valoriser une certaine conception des rapports sociaux de sexe approchant la complémentarité plutôt que l'interchangeabilité, l'expression 'respectueuse des différences' pouvant être interprétée comme une réification et une naturalisation des différences relevant de socialisations différenciées :

Promouvoir une éducation fondée sur le respect mutuel des deux sexes. L'objectif d'élargissement des choix professionnels,

\footnotetext{
${ }^{7}$ Cf. le site : http://www.education.gouv.fr/syst/egalite/conv.htm
} 
au-delà de l'accompagnement des choix d'orientation, exige une action dès le plus jeune âge sur les représentations des rôles respectifs des hommes et des femmes. Il se double d'un aspect plus ambitieux : favoriser une société plus égalitaire et respectueuse des différences.

Or, cette logique sous-jacente est précisément celle qui est présente dans la pensée de la complémentarité entre les sexes, où les attributs sociaux sont naturalisés. Cela permet alors à la panique morale autour de la pédophilie d'obtenir une justification sociale plus importante encore : s'il existe une complémentarité légitime, alors il est raisonnable de s’inquiéter lorsque des hommes remplissent des fonctions pensées comme féminines : câliner un enfant, le changer ou le laver, etc.

On voit donc ici qu'on peut relier le contexte particulier de l'idéologie de la complémentarité des sexes dans l'animation et l'apparition d'une certaine panique morale, dans la mesure où une action réciproque entre ces deux faits s'instaure. D'une part, si l’idéologie de la complémentarité est légitimée, alors dans le cas où une amorce d'interchangeabilité émerge, cela sera critiqué au nom de la panique morale. D’autre part, lorsque la panique morale devient légitime pour «protéger les hommes du soupçon ", alors l'idéologie de la complémentarité n'en est que renforcée. Les enquêté(e)s expliquent les règles diffuses et parfois implicites qui vont consolider à la fois la panique morale et l'idéologie de la complémentarité, renforçant par là l'asymétrie entre animateurs et animatrices face au travail de care.

\section{Le renforcement de l'idéologie de la complémentarité} par la panique morale

\section{Le processus historique d'individualisation de l'enfant et la panique morale actuelle}

Il faut rappeler qu'au terme d'un long processus historique, une nouvelle prise en considération de l'enfance apparaît (Ariès 1960), désignant l'enfant comme une personne à protéger. La dynamique de la modernité va progressivement émanciper l'enfant tout en accentuant sa protection, et l'histoire de l'enfance 
peut être appréciée comme un processus d'autonomisation où celui-ci tend à atteindre le statut d'individu (Renaut 2002). Cette perspective offre le moyen de penser la contradiction entre "la volonté d'éduquer et l'impossibilité de soumettre cet enfant à un pouvoir » (Vigarello 2002). Cette individualisation rend l'enfant détenteur de nouveaux droits, comme celui de la protection, notamment celle de son corps. On peut alors considérer que le concept de panique morale va désigner une angoisse moderne liée à la transgression de ces nouveaux droits. Reprendre la notion de panique morale permet donc de désigner le soupçon généralisé qui va porter sur les hommes travaillant auprès de jeunes enfants, alors que la réalité des transgressions de l'intégrité du corps de l'enfant ne concerne ni uniquement des hommes, ni seulement la sphère professionnelle.

Analysant la situation américaine, Loïc Wacquant montre que la panique morale n'est pas sans fondement étatique ni juridique (2004). Elle n'est pas le fait d'individus déraisonnables et émotifs, elle ne surgit pas de nulle part, mais elle répond à une volonté de répression plutôt que de soin envers les individus désignés comme 'pédophiles'. Alors que l'inceste reste une part majeure de la pédophilie, la panique morale agit à l'encontre des professeurs des écoles, des animateurs et autres professionnels de l'enfance. C'est ce que souligne Loïc Wacquant (id.) :

La focalisation du débat public sur la figure solitaire du pédophile sortant de prison a l'immense avantage de renforcer l'idée reçue selon laquelle la menace criminelle pesant sur les enfants émanerait essentiellement d'individus dépourvus non seulement de moralité mais aussi de toute attache sociale. Et ainsi d'expulser magiquement la violence sexuelle hors de la famille ${ }^{8}$ alors même que toutes les études s'accordent sur le fait que la vaste majorité des atteintes contre les enfants (près de $80 \%$ de celles qui sont enregistrées) sont le fait d'un parent ou d'un proche de la victime, et qu'elles sont étroitement corrélées aux violences contre les femmes. Cette représentation est d'autant plus attractive que la famille patriarcale est soumise à des torsions plus fortes du fait du bouleversement en cours des rapports entre les genres et entre les générations d'une part, et

\footnotetext{
${ }^{8}$ Souligné par l'auteur.
} 
de l'érosion de la sphère domestique par le salariat dérégulé de l'autre.

Il faut néanmoins souligner que si la panique morale semble si présente ici, écartant la famille du soupçon, cela est dû au fait que l'enquête se situe dans ce type particulier d'institution. À l'inverse, dans d'autres institutions telles que les PMI (protection maternelle et infantile), c'est au contraire sur les familles (surtout des classes populaires) que pèse ce soupçon, et non sur l'institution elle-même ${ }^{9}$.

Les chiffres actuels montrent qu'une plus grande judiciarisation des affaires de pédophilie est visible médiatiquement aujourd'hui, les plaintes apparaissant comme plus légitimes et normales, les faits étant reconnus dorénavant comme des crimes qui auparavant ne retenaient pas une attention si soutenue (ou tout du moins extrêmement variable selon les statuts sociaux des personnes impliquées), comme le montre Georges Vigarello (1998). On voit bien le caractère relatif des normes désignant aujourd'hui la maltraitance, qui relèvent de constructions sociales ayant amené peu à peu la société à ne plus tolérer certaines formes de violences, et à les nommer 'déviances' (Gavarini, Petitot 1998). On peut en déduire que la panique morale ne s'établit ni en tout lieu ni en tout temps, mais qu'elle est le fruit concomitant de la construction culturelle de l'enfance, du traitement qui doit être le sien, et des normes de genre établissant les légitimités à s'occuper de l'enfant, faisant de la complémentarité un régime admis comme légitime. L’idéologie de la complémentarité entre les sexes 'prépare le terrain' de la panique morale, dans le sens où elle fige à l'avance ce qui est vu comme comportement normal pour un homme auprès d'un petit enfant, en l'excluant des tâches de care. Agissant en réciprocité, la panique morale renforce le 'bien-fondé' normatif de la complémentarité dans le sens où elle insuffle un sentiment de risque terrible pour celui qui braverait les normes de la complémentarité : celui du soupçon de pédophilie porté sur soi, qui fait honte et culpabilise, au point que les animateurs cherchent à justifier l'évitement de certaines pratiques par un interdit législatif ou moral.

\footnotetext{
${ }^{9}$ Je remercie Numa Murard pour cette précision.
} 


\section{Similitudes et divergences dans la relation enfant/adulte à l'école et au centre de loisirs}

S'interroger sur les différences et les similitudes existant entre l'école maternelle et le centre de loisirs sur ce point permet de comprendre comment s'incarne la panique morale autour de la pédophilie dans ces deux institutions. L'article de Gilles Brougère et Joseph Tobin (2002) relate les réactions de professeurs des écoles et d'élèves en IUFM face à des récits de situations, qui ont déjà été testés auprès d'une population semblable aux États-Unis, où cette inquiétude des adultes en regard de la sexualité enfantine d'une part, et de la crainte de la pédophilie d'autre part, est beaucoup plus criante qu'en France, $a$ priori. L'analyse porte sur les réactions des enseignants lors des entretiens collectifs, car ces histoires "ont été construites pour inciter les enseignants à parler en révélant leurs croyances, valeurs et préoccupations ». L'histoire suivante est la plus proche d'une situation en CLSH :

Dans l'école où je suis la directrice, nous n'avons pas d'enseignants de sexe masculin à plein temps mais il y a un remplaçant qui vient trois fois par semaine. La semaine dernière, à la fin de la journée, il lisait une histoire à ses élèves, et une des petites filles était appuyée contre lui, suçant son pouce, presque comme si elle était allongée sur ses genoux. D’un côté j'étais contente de la voir si détendue et câline avec lui, mais il y avait quelque chose qui me gênait dans la situation. J'aimerais bien qu'il n'en soit pas ainsi, mais je dois reconnaître que j'étais gênée.

La réaction des enseignants américains est la crainte de la pédophilie :

Les hommes apparaissent comme dangereux pour les enfants et vulnérables à l'accusation de pédophilie [...]. Le préscolaire apparaît trop risqué pour les hommes. Mais surgit derrière l'idée qu'il est suspect qu'un homme puisse prendre en charge de jeunes enfants, alors que cela est conforme aux caractéristiques mêmes de la femme.

On retrouve ici, comme chez les animateurs étudiés, la désignation de pratiques spécifiques au care et attribuées selon l’idéologie de la complémentarité, ce qui permet un terrain favorable aux suspicions de pédophilie à l'égard des hommes. D'ailleurs, si les enseignants français critiquent en premier lieu 
la possibilité d'un favoritisme déstabilisant les relations égalitaires entre l'enseignant et tous les enfants (ce qui existe aussi dans l'animation), les animateurs quant à eux recourent plus souvent à la crainte de la pédophilie qu'au problème du favoritisme quand ils commentent une situation de ce type ${ }^{10}$. Or on conseille aux élèves en IUFM « de ne pas être trop proches des enfants, mais indépendamment de leur genre et pour des raisons pédagogiques, qui fonctionnent comme une évacuation de la dimension affective de la relation adulte/enfant en France », ce qui n'est pas du tout le cas des animateurs qui, au contraire, sont encouragés à développer, dans une juste mesure et sans favoritisme, des relations affectives, dont les contours peuvent sembler plus flous que ceux de la relation enseignant/élève.

Cela permet de constater que le regard des enseignants français centré sur leur action pédagogique, les amenant à moins d'attention autour des pratiques sanitaires, se démarque du regard de l'animateur qui est justement plus attentif à ces questions. Les jeunes adultes reçoivent lors du BAFA des rudiments d'apprentissage en psychologie de l'enfant, et les animateurs interrogés justifient souvent leurs pratiques en utilisant des principes éducatifs s'appuyant sur des savoirs psychologiques (Herman 2006) - pouvant de plus naturaliser et légitimer l’idéologie de la complémentarité — de la même façon que les principes éducatifs parentaux relèvent de la psychologisation de l'éducation élaborée autour de la découverte des potentialités de l'enfant (Singly 2003). Or cet autre regard de l'adulte (celui des animateurs et animatrices) se fonde sur ces principes psychologiques sans s'accompagner, comme dans le monde scolaire, d'une visée pédagogique explicite et d'un contenu précis à enseigner, les animateurs étant là exclusivement pour "être proche(s) de l'enfant », et lui "proposer une journée de loisirs éducatifs » (comme l'expliquent les projets pédagogiques des structures étudiées). Cette définition du travail d'animation en CLSH déplace alors le travail de care de la marge au centre de l'action, et donne davantage de vigueur à l'appui sur l'idéologie de la complémentarité, que la panique morale contribue à amplifier.

\footnotetext{
${ }^{10}$ Comme on le voit dans les entretiens formels et informels réalisés avec eux.
} 


\section{Inégalités entre animatrices et animateurs face au travail de care : normes et assignations de genre}

Les animateurs travaillant en maternelle expliquent les difficultés auxquelles ils font face, du fait de l’idéologie de la complémentarité et du contexte de panique morale. Pour Laurence Gavarini et Françoise Petitot (1998) :

Les affaires de pédophilie servent unanimement de repoussoirs. Ce sont précisément ces cruautés et monstruosités qui occupent le devant de la scène, induisant dans le message de prévention une pensée insidieuse : l'intérêt pour l'enfant, 'l'amour des gosses', liés à la position d'éducateur, parent ou professionnel, pourraient bien cacher des désirs sexuels inavoués.

Mathias (22 ans, CLSH maternel et primaire) explique comment cette menace du soupçon de pédophilie a progressivement modifié ses pratiques, comme par exemple celle de changer un enfant. Cet échange entre nous montre le caractère ambivalent de sa décision, c'est autant par crainte du soupçon que pour se décharger sur les animatrices de ces tâches ingrates que Mathias les délègue, car elles sont considérées pour une part comme du 'sale boulot' :

Je n'ai jamais été accompagner une fille aux toilettes, ou je n'ai jamais emmené une fille à l'infirmerie, je n'ai jamais fait ça, parce que justement, à l'époque on m'a dit "non, évite », parce que là, c'est tendu maintenant... J'ai fait « ouais, bah ouais », ça ne me dérangeait pas quoi, à la limite tant mieux, comme ça, ça me laisse plus de temps pour délirer avec tous les enfants... Et du coup les pipis et les cacas de l'époque je disais : "ah s'il te plaît, tu peux t'en occuper ?...»

— À une fille?

Bien sûr... ouais... bien sûr à une fille...

- C'est tout le temps à l'animatrice qu'on demande de changer les gamins... finalement l'animatrice a des tâches qu'elle fait et que l'animateur ne fait pas, est ce que ce n'est pas un peu gênant?

Bah moi je suis tout à fait d'accord avec toi, mais ce n'est pas moi qui vais m'en plaindre...

On remarque d'emblée que la panique morale s'appuyant sur l’idéologie de la complémentarité comme norme légitime va 
renforcer cette logique, permettant ici aux hommes de transformer ce qui était une entrave à l'exercice du métier en une source de pouvoir ${ }^{11}$, en déléguant les pratiques de care. Précisément celles qui sont à la fois les plus ingrates et proches du domaine du sale, donc susceptibles d'être dévalorisantes, et en même temps les plus risquées par rapport au phénomène de panique morale. Les tâches relatives au travail de care deviennent donc progressivement impossibles à déléguer d'une animatrice vers un animateur, alors qu'il semble normal et correct qu'un animateur les délègue à une animatrice.

Cependant certaines animatrices souhaiteraient changer cette répartition. C'est le cas par exemple de Laura qui prévoit, en tant que future adjointe de direction pendant un mois d'été chez les tout-petits (2/3 ans), d'imposer à tous, animateurs et animatrices, de changer les enfants. Elle précise que les garçons comme les filles doivent prendre d'extrêmes précautions quand ils changent ou habillent un enfant, c'est-à-dire qu'ils doivent s'abstenir presque totalement de toucher l'enfant, mettant en œuvre des stratégies que Laura leur délivrera. Cela permet donc d'entrevoir le projet d'interchangeabilité que souhaite Laura sur ce point précis (changer l'enfant), alors qu'elle reste attachée à une vision naturalisée des rôles sexués. Cependant, comme on va le voir, racontant spontanément sa manière d'agir quand elle change un enfant, son récit fait intervenir directement 'une animatrice', et non l'appellation au masculin neutre qui pourrait révéler un appui égal sur les hommes comme sur les femmes pour la seconder dans ces tâches particulières.

- Mais toi personnellement, en temps qu'adjointe, tu diras aux mecs de ne pas changer les gamins ?

Bien sûr que si, ils les changent, ah si! Moi je pars de ce principe...

- Mais ça peut aussi leur permettre d'essayer de se décharger. J'entends des garçons, quand je leur pose ces questions-là, me dire : "Oui bah c'est vrai que déjà il y a tout ce contexte de pédophilie, qui fait qu'on est vite suspecté en temps que garçon, qu'on n'est pas à l'aise et tout. » Mais ils me disent aussi :

\footnotetext{
${ }^{11}$ Merci à Danièle Kergoat pour cette remarque explicitant clairement ce qui était suggéré.
} 
"Moi je t'avoue concrètement que ça ne me fait pas de mal de m'en décharger quoi, parce que ce n'est pas un truc que j'aime..."

Ouais. Alors moi je vais te dire tout de suite, déjà moi quand je change un enfant, je ne suis jamais toute seule avec lui, que ce soit de sexe féminin ou masculin, quand c'est un bout de chou... Deuxièmement, je ne le touche pas, je n'ai jamais touché un enfant pour le changer! Alors là on me demande : "Comment tu fais? Parce que si tu changes un enfant, est-ce que tu le laves, tu ne le laves pas? " Alors moi je t'explique pour la Colline [...]. Je demande à une animatrice en temps normal qu'elle vienne avec moi [...]. Et c'est l'enfant qui se découvre complètement tout seul. À partir du moment où moi j'ai juste enfilé les pieds [du pantalon], il se débrouille, et je fais en sorte qu'il y ait toujours l'animatrice d'à côté qui soit présente, et pourtant je suis une femme! Parce qu'il y a tout ce contexte, là, où les enfants... Ouais, ce n'est pas méchant quoi ! [...] Et maintenant pour en venir aux garçons, ce n'est pas parce que tu es un garçon que tu n'as pas le droit de changer un enfant, à partir du moment où tu le fais euh... tu le fais, tu trouves la technique où tu te sens le plus à l'aise.

Juste avant d'expliquer ses méthodes, Laura avait détaillé son point de vue quant à l'organisation des animateurs et animatrices au centre de loisirs. Cela permet de remarquer que l'argument de la panique morale n'apparaît qu'après avoir rappelé les standards de la complémentarité, ce qui est fréquent dans les récits recueillis. Et, finalement, ce n'est pas tant la difficulté rencontrée par les hommes pour changer les enfants qui semble, selon Laura, l'explication. Pour elle, c'est davantage le « besoin de bouger » des hommes animateurs qui explique leur moindre demande d'être recrutés en maternelle. Elle revient donc à une image naturalisant les comportements attendus des hommes et des femmes, tout en décrivant la différence physique qui pourrait gêner l'enfant.

- Et au niveau du fait qu'en maternelle ce soit la plupart du temps une majorité d'animatrices par rapport aux garçons, enfin il y a des équipes tout le temps plus féminines, toi, comment tu l'expliques? Le fait que les animatrices soient beaucoup plus présentes en maternelle qu'en primaire ? Et est-ce que tu trouves ça bien ?

Alors, moi je pars du principe que... je ne trouve pas ça spécialement bien, mais je pense à mon sens que... euh... ça... la 
présence féminine elle est plus ou moins importante parce qu'en fait l'enfant il a besoin, il est tout petit, petit, petit, c'est presque, c'est encore considéré comme... je ne sais pas si c'est un nourrisson ou pas, mais c'est un tout petit bout'chou...

Et c'est vrai, que nous déjà les femmes dans notre physique, on n'a pas la même corpulence qu'un homme. Je veux dire, si tu mets Alexis, on voit tous très bien comment il est physiquement, mais je vais un peu le décrire ça va t'aider, et pour ton [mémoire]... Voilà, euh, 1,80 m, tout en muscles, grand black, super sportif, les bras bien costauds... Et «Bonjour, je m'appelle Axelle, j'ai deux ans et demi et je dois faire je sais pas combien de centimètres... je pèse quoi... cinq kilos, on peut faire ses bras avec mon corps... »12.

Ce n'est pas super évident quoi... Ça ne rassure pas l'enfant. Je ne dis pas qu'il faut un physique particulier, loin de là... Mais si tu veux, la dominante féminine dans un centre de loisirs, déjà que l'enfant il n'a pas sa mère [...]. Maintenant, j'ai envie aussi de te dire que les animateurs ils ont envie de bouger, un garçon au BAFA, qui s'occupe des deux ans et demi, il n'a pas envie de changer les pipis, les cacas parce que, il a envie de bouger, lui aussi l'air de rien c'est quelqu'un d'actif ! [...] Et aussi je pense que ces dernières années, c'est tout ce qu'il y autour de la pédophilie...

Si le soupçon de pédophilie peut aussi toucher les femmes qui s'occupent des jeunes enfants, on assiste également à une 'protection' des hommes face à ce soupçon, conduisant les animatrices à s'y exposer davantage, tout en se conformant aux stéréotypes de sexe propres à l'idéologie de la complémentarité. Sonia (25 ans, animatrice en maternelle et primaire depuis six ans) explique ainsi comment la décision de ne laisser que les femmes changer les enfants fut prise à la Colline, lorsque je lui demande quels sont les moments où la "différence fille/garçon » entre en considération :

Alexis, je crois, m'a dit : " bon bah elle, il faut que tu l'emmènes aux toilettes, moi je ne peux pas l'emmener quoi...». Dans ce cas-là, le côté fille/garçon, c'était important... Mais sinon... en dehors de ces moments-là... C'est vrai que moi j'avais plus ten-

\footnotetext{
${ }^{12}$ Laura veut dire que le corps d'Alexis est démeusuré par rapport à celui d'Axelle et que la 'masse' de ses bras correspond à celle du corps de la petite fille.
} 
dance à jouer l'infirmière ou changer les enfants que Alexis... Parce que Alexis... On était un peu tous les deux dans le même esprit, que, c'était pour protéger les animateurs hommes, c'est mieux que ça soit une femme qui change les enfants quoi... C'est pas forcément bien, mais ça permettait la protection des animateurs... Pour qu'il n'y ait pas de problème de suspicion ou quoi que ce soit...

Une asymétrie et une inégalité entre animatrices et animateurs s'installent donc par rapport au travail de care, en étant rarement contestées sur les terrains observés. S’il est arrivé que certaines animatrices (seulement deux) critiquent cette répartition en réunion, l'argumentation autour du risque de suspicion encouru par les hommes a suffi à clore la discussion, et à renforcer la légitimité de la complémentarité. Alors que les tâches professionnelles officiellement prescrites sont les mêmes pour tous, cette version de la croyance en "l'égalité dans la différence » permet donc de prolonger l'inégalité entre animateurs et animatrices face au travail de care en CLSH maternel, car dans cette croyance :

L'objectif est d'établir un rapport de nécessité réciproque entre les deux groupes, réciprocité qui produirait à son tour l'égalité » [en passant par] "la construction pour les femmes d'un domaine d'expertise qui leur soit réservé et leur garantisse une place spéciale et indispensable à la société. [...] La mise en place d'un métier : la maternité (Delphy 2001).

La panique morale joue ici le rôle de justification et renforce l’idée selon laquelle, pour les jeunes femmes, l'expérience de l'animation en centres de loisirs maternels constitue une initiation aux normes de la maternité. Il devient alors doublement légitime de se fonder sur la panique morale pour investir l’idéologie de la complémentarité : d'une part, pour protéger les hommes du soupçon et les éloigner du travail de care et du domaine du sale et, d'autre part, pour se conformer au déploiement dans « le secteur public et marchand de ce développement de l'enfance comme domaine d'expertise et d'action [...], non pas en rivalité avec les femmes, mais pour les servir et surtout les guider et les transformer en vraies mères » (id.). Dans le cadre de l'animation, les jeunes adultes s'occupant des enfants ne servent pas littéralement de guides aux mères, mais l'incarnation d'un rôle 
affectif et éducatif envers l'enfant les amène à se considérer dans des rôles proches de ceux des parents, où les rôles sont sexués. Expliquant cela, ils rappellent toujours l'injonction qui leur est faite de rester dans une place définie et de ne surtout pas "se prendre pour les parents ", mais certains voient dans l'expérience de l'animation une 'préparation' au travail de parent. L’idéologie de la complémentarité renforcée par la panique morale permet alors de faire passer ce qui est une inégalité en une nécessité pour la protection de l'enfant et pour le développement d'identités sexuées où la maternité devient un prolongement logique. D'autre part, cela permet de masquer le fait que «la sollicitude, l'aptitude à se soucier des autres [...] n'est pas une disposition morale spécifique aux individus de sexe féminin mais qu'elle est clairement l'effet d'une position subalterne: en un mot, le care n'est pas tant une morale genrée qu'une morale sociale, une disposition éthique liée au statut de dominé-e-s » (Dorlin 2005).

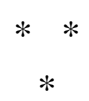

On a pu voir les difficultés qui se posent dans l'animation quant à l'émergence d'équipes professionnelles totalement mixtes, dans ce climat de panique morale, où la menace du soupçon de pédophilie portée sur les hommes s'établit d'autant plus facilement qu'une idéologie de la complémentarité des tâches différenciées des animateurs (jeu et autorité) et animatrices (soin et affection) semble prégnante dans l'animation. Il est important de poser le problème de la panique morale comme construit social, non pas seulement au niveau de la réalité de la menace planant sur les personnes, mais aussi de la question des faits sociaux qui sont produits par ce contexte. Ainsi nous avons pu voir comment, dans un contexte de panique morale, l'idéologie de la complémentarité agit en faisant apparaître a priori une 'normalité' selon laquelle les hommes ne doivent pas (trop) approcher le corps de l'enfant, qui ne peut alors qu'être renforcée et acquérir une nouvelle légitimité. Le travail de care devient alors d'autant plus un enjeu de hiérarchisation (où le domaine du sale et du soupçon incombe aux femmes pour que les hommes en soient protégés), et d'assignation au travail 
symboliquement tourné vers 'la maternité'. Ceci nous permet de penser, avec Peter Berger et Thomas Luckmann (1986) que les moments de reconstruction identitaire font place à des oppositions du type "Avant je croyais que " / "Maintenant je sais que », qui sont l'occasion de la dramatisation d'un changement effectif. En effet, Loïc Wacquant le soulignait d'ailleurs, c'est au moment précis d'une reconstruction des identités de genre, où il deviendrait également légitime pour l'homme comme pour la femme de pratiquer n'importe quelle activité, y compris les activités de care ou de maternage, que surgit dans les médias, dans le droit et dans 'l'opinion publique' (en utilisant avec précaution cette expression) cette crainte de la pédophilie, qui n’a jamais été aussi vive, et aussi émotionnellement chargée, et qui est utilisée ici pour temporiser ou contrebalancer cette recomposition. Si cela ne doit pas masquer la réalité d'une plus grande considération accordée à l'enfant en tant que personne possédant des droits, permettant une plus grande vigilance des adultes face à la pédophilie, davantage punie et médiatisée aujourd'hui qu'hier, il convient d'examiner les conditions dans lesquelles se réalise le travail de care autour de l'enfant en centres de loisirs maternels, et de constater les inégalités entraînées par le recours à l'idéologie de la complémentarité légitimée par la panique morale.

\section{Références}

Ariès Philippe (1960). L'enfant et la vie familiale sous l'Ancien Régime. Paris, Plon «L'univers historique ».

Bacou Magali (2004). «La mixité sexuée dans l’animation ». Agora débats/jeunesse, $\mathrm{n}^{\circ} 36$.

Berger Peter, Luckmann Thomas (1986). La construction sociale de la réalité. Paris, Armand Colin « Sociétés » [1 ${ }^{\mathrm{re}}$ éd. 1966].

Bessin Marc (2005). «Le travail social est-il féminin ?». In Ion Jacques (ed). Le travail social en débat[s]. Paris, La Découverte «Alternatives sociales ».

Brougère Gilles, Tobin Joseph (2002). « Culture et sexualité enfantine à l'école maternelle. Étude comparée entre les États-Unis et la France ». Éducation et sociétés, vol. 2, $n^{\circ} 6$. 
Cohen Stanley (1972). Folk Devils and Moral Panics: The Creation of Mods and Rockers. London, MacGibbon \& Kee.

Delphy Christine (2001). L'ennemi principal. Tome 2. Penser le genre. Paris, Syllepse « Nouvelles questions féministes ».

Dorlin Elsa (2005). « Dark Care : de la servitude à la sollicitude ». In Paperman Patricia, Laugier Sandra (eds). Le souci des autres. Éthique et politique du care. Paris, EHESS « Raisons pratiques ».

Gavarini Laurence, Petitot Françoise (1998). La fabrique de l'enfant maltraité. Un autre regard sur l'enfant et la famille. Paris, Érès.

Goode Erich, Ben-Yehuda Nachman (1994). Moral Panics. The Social Construction of Deviance. Cambridge, USA, Blakwell.

Herman Elisa (2006). " Les normes de genre et d'autonomie dans la socialisation. Enquête sociologique auprès d'animateurs en centres de loisirs ». Mémoire de master 2 recherche, Université Paris 5.

Lebon Francis (2005). Une politique de l'enfance. Du patronage au centre de loisirs. Paris, L'Harmattan « Logiques sociales ».

Renaut Alain (2002). La Libération des enfants. Contribution philosophique à une histoire de l'enfance. Paris, Bayard.

Rousseau Michel (1997). Les emplois de l'animation en Île-de-France. Étude prospective régionale 1995-1997. Paris, La Documentation française.

Singly (de) François (2003). Les uns avec les autres. Quand l'individualisme crée du lien. Paris, Armand Colin «Individu et société ».

Vigarello Georges (1998). Histoire du viol, $X V I^{e}-X X^{e}$. Paris, Seuil « L'univers historique ».

- (2002). «Les paradigmes d'une histoire de l'enfance ». Le Débat, $\mathrm{n}^{\circ} 121$.

Wacquant Loïc (2004). Punir les pauvres. Le nouveau gouvernement de l'insécurité sociale. Marseille, Agone « Contre-feux ». 